\title{
Evasive Mechanisms in Cancer Cells Pose Challenges to Effective Therapy and Prevention of Cancer Recurrence
}

\author{
Celestial T. Yap ${ }^{1,2^{*}}$ \\ ${ }^{1}$ Department of Physiology, Yong Loo Lin School of Medicine, National University of Singapore, Block MD9, 2 Medical Drive, Singapore 117597 \\ ${ }^{2}$ National University Cancer Institute Singapore (NCIS), 1E Kent Ridge Road, NUHS Tower Block Level 7, Singapore 119228
}

*Corresponding author: Celestial T. Yap, Department of Physiology, Yong Loo Lin School of Medicine, National University of Singapore, Block MD9, 2 Medical Drive, Singapore 117597, Tel: 6516 3294/ 8513; Fax: 67788161; E-mail: celestial_therese_yap@nuhs.edu.sg

Received: March 04, 2014; Accepted: March 06, 2014; Published: March 08, 2014

Copyright: (c) 2014 Yap CT. This is an open-access article distributed under the terms of the Creative Commons Attribution License, which permits unrestricted use, distribution, and reproduction in any medium, provided the original author and source are credited.

\section{Editorial}

The exponential gain in knowledge and understanding of cancer biology, as well as cumulative experience from clinical disciplines involved in cancer treatment in the last few decades have resulted in new therapeutic options for several types of cancer. Whilst wellestablished chemotherapeutic drugs remain the drugs of choice for many cancers, they affect cellular functions non-selectively throughout body tissues by their common effects on fundamental mechanisms underlying cell division and cell death. More recently, targeted therapy against subtypes of tumours which bear distinct molecular signatures are now available, and may be used in combination with chemotherapy.

Targeted therapy has been demonstrated to be useful in treating several cancers, including breast, colon and gastric cancers. For example, anti-estrogen therapy has been the mainstay of treatment for Estrogen Receptor (ER) - positive breast cancers which are hormonesensitive, whilst breast cancers which over express the Human Epidermal Receptor 2 (HER2) can now be effectively treated with drugs that target HER2, including the antibody-based drug, trastuzumab. HER2 is a member of the Epidermal Growth Factor Receptor (EGFR) family which comprises tyrosine receptor kinases involved in signalling cascades that contribute to cancer growth and spread, by promoting cellular proliferation and invasion. Another example of targeted therapy that is being explored include drugs which inhibit the cMet-Hepatocyte Growth Factor (HGF) pathway - this is amplified in a variety of solid cancers and transforms cancer cells into more aggressively proliferating, invading and cell death-resistant phenotypes.

However, major challenges to the control of cancer spread and recurrence remain unresolved - it is now known that tumour cells engage evasive mechanisms to 'out-smart' the onslaught of aggressive treatment. In fact, the 'plasticity' of cancer cells has been suggested to resemble accelerated evolution in the tendency towards the 'survival of the fittest'. This ensures that although most cancer cells are susceptible to chemotherapy and/or targeted therapy, subsets of cancer cells survive that may repopulate the original tumour site or metastasize to distant sites. These subsets remain, often as a result of various survival mechanisms that are activated in response to drugs, such as recruitment and amplification of alternative signalling pathways that promote survival and prevent apoptosis, including Phosphoinositide 3 (PI3)-kinase/Akt, Src kinases and Sphingosine Kinase 1 (SphK1). The heterogeneity of tumours arising from misregulated DNA replication also favours the accumulation of mutations which render cells relatively resistant to apoptosis under stressful conditions. In addition, alterations in epigenetic regulation of gene expression in response to cellular stress may lead to the suppression of pathways that cause cell death, and re-emergence of those that promote cell survival. Recent evidence has revealed yet another novel mechanism that may contribute to survival of cancer cells - autophagy (in Greek, meaning 'self-eating'). Autophagy enables cells to degrade proteins and organelles, releasing substrates that can be recycled and re-used to cope with stress. However, autophagy can also result in cell death, and it is unclear how cancer cells regulate this mechanism for survival.

A subset of tumour cells that is relatively chemo resistant and is of increasing interest as being potentially targetable is the cancer stem cell, which has the potential to recapitulate the development of a tumour mass. The complexity of cancer treatment is further exemplified when one considers the tumour microenvironment, where interactions with other non-tumour cells (e.g. macrophages and fibroblasts) may promote tumour growth and spread. It is thus clear that multiple strategies are required to counteract the evasive nature of cancer cells. Ideally, diagnostic tools which monitor the evolution of the cancer within the patient and identify critical changes that signal the need to change treatment strategies could also be developed.
This article was originally published in a special issue, entitled: "Trends in Antimicrobial Agents and Chemotherapy", Edited by Syreeta L. Tilghman, University of Louisiana, USA 